\title{
Application of neuromuscular electrical stimulation of the lower limb skeletal muscles in the rehabilitation of patients with chronic heart failure and chronic obstructive pulmonary disease
}

EWA BARBARA KUCIO1, B, D-F, JUSTYNA NIESPOREK ${ }^{1,8, \text { E, F, CEZARY KUCIO }}$, 2, в, D-F

${ }^{1}$ The Department of Physiotherapy at the Jerzy Kukuczka Academy of Physical Education in Katowice

${ }^{2}$ The Multidisciplinary Hospital in Jaworzno

A - Study Design, B - Data Collection, C - Statistical Analysis, D - Data Interpretation, E - Manuscript Preparation, F - Literature Search, G - Funds Collection

Summary Increasing physical activity is a widely-known method of rehabilitation of patients with chronic heart failure (CHF) and chronic obstructive pulmonary disease (COPD). However, what kind of procedure is to be applied if a patient suffers from advanced heart or respiratory failure, cannot undertake physical exercise due to locomotor system disorders or is currently undergoing respiratorotherapy? Recent research shows that neuromuscular electrical stimulation of the lower limb skeletal muscles (NMES) may comprise an alternative to physical training in patients with CHF and COPD. The aim of this study is to summarize the current state of knowledge on the use of NMES in cardiac rehabilitation of patients with CHF and pulmonary rehabilitation of patients with COPD. As demonstrated in recent research on the topic, NMES - due to forcing the muscles to activate - increases exercise tolerance, muscle mass and endurance in patients with CHF and COPD. The beneficial effect of NMES on blood circulation in the muscles, aerobic enzymes activity, functioning of the vascular endothelium, reduction of pro-inflammatory cytokines concentration and increased quality of life has also been presented. It is to be accentuated that NMES treatment, due to lesser physical exertion and, in turn, a decreased feeling of dyspnea are more comfortable for the patient than traditional physical training. Moreover, NMES treatment, after foregoing training, can be applied at home. Potential side effects include transient muscle pain and minor skin damage due to improper positioning of the electrodes. To summarize, NMES treatment is well received by CHF and COPD patients and brings about increased exercise tolerance, as well as better quality of life. Devices used for NMES therapy, due to progressive miniaturization, are easily accessible and relatively inexpensive.

Key words: neuromuscular electrical stimulation, chronic heart failure, chronic obstructive pulmonary disease.

Kucio EB, Niesporek J, Kucio C. Application of neuromuscular electrical stimulation of the lower limb skeletal muscles in the rehabilitation of patients with chronic heart failure and chronic obstructive pulmonary disease. Fam Med Prim Care Rev 2017; 19(1): 71-74, doi: 10.5114/fmpcr.2017.65095.

\section{Background}

Chronic heart failure (CHF) and chronic obstructive pulmonary disease (COPD) have become a valid health problem on a worldwide scale. The primary clinical symptoms of both $\mathrm{CHF}$ and COPD, regardless of organ localization, etiology and pathophysiology are a progressive decrease of exercise tolerance due to the occurrence of dyspnea and fatigue during exercise. In the first stage of the disease, these symptoms occur mainly during strenuous physical exercise. As the disease progresses, the symptoms begin to manifest during everyday activities, which results in decreased self-reliance of the patients. Therefore, $\mathrm{CHF}$ and COPD constitute a problem reaching far beyond the health aspect, as the diseases affect the patients in their social functioning, bringing about disability, lower quality of life and social isolation. These factors, in turn, result in an increased number of hospitalizations, which requires additional financial expenditure.

Recently, in numerous publications, it has been suggested that the application of physical training in the rehabilitation of patients with CHF and COPD is safe and beneficial, with increased physical endurance, as well increased oxygen consumption at the peak of physical exertion, among the main benefits $[1,2]$. Physical training also increases the effects of ventilation.
This indicator may depend on decreased ergoreceptor activity in the skeletal muscles and increased baroreceptor activity during physical training [3]. Simultaneously, capillary placenta enlargement in the skeletal muscles during physical training was observed [4].

Physical training not only increases physical tolerance, but also brings about a better quality of life, which is directly correlated with health $[5,6]$. The patients observe increased physical fitness, mental well-being and perform their daily activities with greater ease, which also results in a feeling of physical independence and makes the symptoms of the disease less apparent. However, it needs to be accentuated that in order for long-term benefits of the therapy to manifest, the physical training and therapy ought to become the patients' lasting lifestyle change.

Allowedly, many patients with $\mathrm{CHF}$ and COPD also suffer from locomotor disorders, which impede physical activity. Moreover, patients with CHF and COPD cannot perform physical training due to advanced or exacerbated heart or pulmonary failure. At times, patients also abort training due to discomfort related to dyspnea.

Neuromuscular electrical stimulation (NMES) of skeletal muscles consists in the application of an electrical current of adequate characterization on skeletal muscles in order to invoke specific reactions, such as: 
- increase in strength of a muscle,

- $\quad$ strengthening the muscle post-surgery or preventing muscle atrophy or rebuilding the strength of a muscle in cases of vast atrophy,

- re-education of motor control of a muscle,

- gaining or increasing range of motion of a joint,

- increasing muscle endurance,

- changes in structure and function of a muscle.

Since the introduction of NMES in clinical practice, researchers have been looking for methods of application of this therapy. Modern technology enables better choice and control of stimulation parameters, and the devices become progressively smaller, less expensive and more mobile. Therefore, increasingly more research is being conducted on the application of NMES in various diseases.

As suggested by current research, NMES of the skeletal muscles of the lower limbs may provide a considerable alternative to traditional physical training in patients with CHF and COPD in periods of exacerbation of the disease or in patients with locomotor disorders who cannot undertake traditional movement activities $[7,8]$.

\section{Application of NMES in patients with chronic heart failure}

Research on the application of NMES in patients with chronic heart failure was conducted on individuals with decreased left ventricular ejection fraction with class II or class III NYHA symptoms. In rare cases, patients with class IV symptoms also participated in the research. Some of the participants were either after a heart transplant or qualified for heart transplant surgery [9-20].

The research demonstrated that the application of NMES in patients with CHF results in improvement of exercise tolerance indicators, such as: increased peak oxygen consumption, increased anaerobic threshold, increased duration of exercise tests and greater distance covered during the 6MWT test [9-20]. After a series of NMES treatment sessions, increased muscle endurance was also noted in patients with CHF [12]. The strength of stimulated muscles increased by approx. $11-20 \%$ in relation to the starting strength of the muscle. The observed improvement of muscular strength applies to both isokinetic $[14,21,22]$ and isometric [21] contractions. It seems that these changes are caused by the increase in aerobic enzyme activity of skeletal muscles, which, in turn, leads to an increased oxidative capacity $[9,12-14,18]$. Moreover, it was observed through the biopsy procedure that due to the application of NMES, an increase in type I muscle fibers occurs, which proves the evident recalibration of the stimulated muscles in the direction of aerobic metabolism and endurance training [13].

Increased blood circulation in the stimulated muscle is another effect of NMES [14]. This phenomenon may be related to the improvement of the anti-inflammatory IL-10 cytokine to pro-inflammatory TNF cytokine relation observed after NMES therapy, which may bring about beneficial, anti-inflammatory effects in patients. Additionally, due to NMES treatment, a significantly reduced number of distilled forms of adhesive molecules, such as: intercellular adhesion molecule (sICAM-1) and vascular cell adhesion molecule (sVCAM-1), occur as "end products" of the interaction between active monocytes and endothelial cells. As suggested by researchers, NMES is a method of training that improves the endothelial function and increases exercise tolerance in patients with CHF, thus breaking the vicious cycle between improper peripheral inflammatory responses, nitric oxide metabolism anomalies and vasculo-muscular dysfunction [9].

It is to be emphasized that neither increased concentration of lactic acid nor increased activity of lactic dehydrogenase are observed in the stimulated muscles [12]. On the other hand, Dobsak et al. [15] have noted a minor, statistically insignificant increase in creatine kinase and lactic dehydrogenase levels in patients with advanced heart failure (with IV class NYHA symptoms) after the first week of stimulation. These changes, however, are suggested to be a reflection of the greater strain put on untrained muscles in the beginning phase of NMES training, similar to reactions taking place in healthy individuals during physical exercise.

In the conducted research, large muscle groups of the lower limbs were stimulated. Simultaneous electrical stimulation of the quadriceps and gastrocnemius muscles was performed most frequently $[9,14,18,20,21]$. Simultaneous stimulation of the quadriceps and the ischiotibial muscles $[13,22]$ or stimulation of the quadriceps muscle exclusively was performed less often $[11,21]$.

In all of the studied cases, an electric current of less than $50 \mathrm{~Hz}$ was used, which is in accordance with the rules of performing endurance training. Endurance training primarily affects the slow-twitch, aerobic muscle fibers in patients with CHF. The following values of electric current were used: $4 \mathrm{~Hz}$ [19], 10 $\mathrm{Hz}[10,14,16,20], 15 \mathrm{~Hz}[13], 25 \mathrm{~Hz}[9,12,17,18]$ and $50 \mathrm{~Hz}$ $[21,22]$.

As far as endurance training is concerned, it is generally advised to perform short muscle contractions for high repetitions. The contraction is then followed by a brief relaxation lasting just as long as the contraction or 2-3 times longer. In patients with $\mathrm{CHF}$, the duration of muscle contraction was short and amounted to approx. 2 seconds $[13,21,22]$ or $5-6$ seconds [9, $12,17]$ with contractions lasting about 10 seconds [11] or even 20 seconds $[14,15]$ being used less often. The relaxation period lasted as long as the contraction or 2-3 times longer at most $[13,21,22]$. The treatment sessions were performed with varying frequency - at least 5 times a week [9, 16-19, 21, 22], up to 7 times a week in some cases $[13-15,20]$. A case was also noted in which 2 sessions a day were performed [20] and one in which the sessions were relatively less frequent -3 times a week [11].

In two of the conducted meta-analyses, in which electric stimulation of the lower limbs and physical training using a cycle ergometer or "supposed" electric stimulation on specific exercise tolerance indicators in patients with $\mathrm{CHF}$ were compared, it was demonstrated that a lesser increase in peak oxygen consumption $\left(\mathrm{VO}_{2 \text { peak }}\right)$, distance covered in the 6MWT test and strength of thigh muscle contraction resulted from applying NMES in comparison to physical training. However, when compared to "supposed" electric stimulation, NMES leads to a greater increase of the abovementioned exercise tolerance indicators. The results of both of the meta-analyses suggest that NMES brings about improvement of exercise tolerance in patients with $\mathrm{CHF}$, although not as significant as the improvement resulting from traditional physical training $[23,24]$.

The conducted research also showed that the combination of classical cardiac rehabilitation (based on physical training) and NMES of skeletal muscles of the lower limbs does not bring about significant improvements of exercise tolerance in patients with $\mathrm{CHF}$ in comparison with traditional rehabilitation [20]. These results may suggest that physical training is the most important element of cardiac rehabilitation. Moreover, a conclusion can be drawn that NMES can be used as an alternative form of rehabilitation in patients with advanced CHF (with class IV NYHA symptoms) or in patients with CHF who cannot perform physical training due to disorders of the locomotor or nervous system.

\section{Application of NMES in patients with chronic obstructive pulmonary disease}

NMES of the lower limb skeletal muscles in patients with COPD, as in the case of patients with CHF, is a relatively new method of rehabilitation. NMES activates the muscles, forcing them to contract, thus causing lower metabolic demand, which puts less strain on the cardiovascular system than random exercise. This, in turn, causes lower ventilation demand, which 
reduces the risk of dyspnea. Patients with advanced COPD, as well as patients suffering from temporary exacerbation of the disease, experience intensified respiratory symptoms, such as: dyspnea, coughing and decreased exercise tolerance, which are one of the main factors causing the progression of the disease. Exacerbation of the symptoms is also the main cause for aborting physical training. Conventional training may be contraindicated during exacerbation periods due to destabilization of the respiratory system, decompensation of the circulatory system and other dangerous clinical symptoms. In previous research using electrical stimulation, it was proven that NMES is safe for patients experiencing exacerbation of the disease, patients with advanced COPD and even for patients undergoing respiratorotherapy [7].

Another important aspect of application of NMES is the reduction of anxiety experienced by the patients during and after NMES treatment in comparison with traditional training sessions. The reduction of anxiety enables the patients to break the emotional barrier and eventually the spiral of inactivity, which, in advanced cases of COPD, often leads to disability or even death.

The number of clinical studies conducted heretofore is not sufficient for unanimous conclusions to be drawn as far as the role of NMES in pulmonological rehabilitation of patients with COPD is concerned. However, the demonstrated results provide a basis for implementing NMES in clinical practice in patients with COPD [25-32].

The presented results do not allow for one particular protocol of NMES of skeletal muscles in patients with COPD to be created. Assuming one uniform protocol for all cases of patients with COPD remains impossible, as various electric current values tailored to the specific needs and reactions of a particular patient need be determined in each case. Nevertheless, an outline of a general NMES protocol has emerged. The treatment sessions were focused primarily on large muscle groups of the lower body, in most cases the quadriceps muscles of both legs $[25,28-30]$ or, simultaneously, the quadriceps and the triceps of the calf [26, 32], the quadriceps and the glute muscles [27], the quadriceps and the popliteus muscle [26, 31].

Biphasic, symmetric impulses lasting from 0.3 to $0.4 \mathrm{~ms}$, appearing at a frequency of 35 and $50 \mathrm{~Hz}$, were preferred. These values were well tolerated by the patients. A minor span of duration of a single impulse was used in the research. Impulses ranging from 300-400 $\mu$ s are recommended for stimulation of large muscle groups, such as the quadriceps or the gastrocnemius muscles. In the presented research, an electric current of $50 \mathrm{~Hz}[25,26,28]$ and $35 \mathrm{~Hz}$ [27] was used. One of the studies did not specify the value of the current applied [29]. The aim of using these specific values was to change the muscle phenotype through achieving maximal activation of slow-twitch, fatigueresistant muscle fibers.
Transient contractions of the muscles were evoked using high repetitions in accordance with the rule in which the duration of the contraction in relation to the relaxation of the muscle ought to be similar to the one occurring during classical endurance training. Following a series of contractions, a pause adjusted to the level of fatigue of the patient occurred. A study by Neder et al. [25], who applied a 2/8 second ratio for 15 minutes during the first week, a $5 / 25$ second ratio for 30 minutes during the second week and a 10/30 second ratio for 30 minutes during the third and following weeks, served as a model for other authors. However, the acquired data does not point to which of the abovementioned ratios of contraction and relaxation is optimal for reaching the desired effect without causing excessive fatigue of the muscles.

A single session lasted, respectively: 15 minutes [25, 26], 25 minutes [28], 30 minutes [25, 26, 27, 29], 35 minutes [28] and 60 minutes [26]. The sessions were usually performed 5 [25-29] or 4 [7] times a week. The total time of NMES application amounted to 4, 5 or 6 weeks [25-32]. The value of electric current used depended on the specific tolerance of the patient. The aim was to achieve apparent, clearly perceptible contraction of the muscles.

Taking into account that about $80 \%$ of patients with an advanced stage of COPD suffer from muscle atrophy, resulting in decreased exercise tolerance, electric stimulation may serve as a method subsidiary to physical training, especially useful during periods of exacerbation. The possibility to regain and develop strength or endurance (depending on needs) with the use of this method may result in increased exercise tolerance. Moreover, a decreased feeling of dyspnea and fatigue is proven to be another benefit of electrical stimulation. In three $[25,26,28]$ of the eight previous studies, electric stimulation did not result in increased exercise tolerance, as opposed to the other five. Only one of the studies linked NMES to pulmonological rehabilitation [29].

\section{Summary}

To sum up the information on the positive effects of applying NMES treatment in patients with CHF and COPD, it can be assumed that NMES causes changes in exercise tolerance similar to those related to traditional physical training. However, it is to be accentuated that NMES training requires less strict medical supervision and can even be performed by the patients at home. NMES is well tolerated by patients, and the devices used for NMES treatment are inexpensive and easily available. No pain, skin damage or exacerbation of current health conditions were noted during NMES treatment. No side effects, such as disruptions of blood pressure or heart rate, were also observed during treatment. Furthermore, NMES does not cause excessive fatigue or damage of the muscle fibers.

Source of funding: This work was funded by the Department of Physiotherapy at the Jerzy Kukuczka Academy of Physical Education in Katowice.

Conflict of interest: The authors declare no conflict of interests.

\section{References}

1. Da Silva MS, Bocchi EA, Guimaraes GV, et al. Benefits of exercise training in the treatment of heart failure. Study with a control group. Arq Bras Cardiol 2002; 79(4): 357-362.

2. Bolton CE, Bevan-Smith EF, Blakey JD, et al. British Thoracic Society guideline on pulmonary rehabilitation in adults. Thorax 2013; 68: ii1-ii30, doi:10.1136/thoraxjnl-2013-203808.

3. Ponikowski PP, Chua TP, Francis DP, et al. Muscle ergoreceptor overactivity reflects detorioration In clinical status and cadiorespiratory reflex control in chronic heart failure. Circulation 2001; 104(19): 2324-2330.

4. Guazzi M, Reina G, Tumminello G, et al. Exercise ventilation inefficiency and cardiovascular mortality in heart failure: the critical independent prognostic value of the arterial $\mathrm{CO}_{2}$ partial pressure. Eur Heart J 2005; 26: 472-480.

5. Flynn KE, Pina IL, Whellan DJ, et al. Effects of exercise training on health status in patients with chronic heart failure. HF-ACTION randomized controlled trial. JAMA 2009; 301(14): 1451-1459.

6. Puhan MA, Buschinh G, Schunemann HJ, et al. Interval versus continuous high-intensity exercise in chronic obstructive pulmonary disease: a randomized trial. Ann Intern Med 2006; 145(11): 816-825. 
7. Sillen MJ, Speksnijder CM, Eterman RA, et al. Effects of neuromuscular electrical stimulation of muscles of ambulation in patients with chronic heart failure or COPD. A systematic review of the English-language literature. Chest 2009; 136(1): 44-61.

8. Banerjee P. Electrical muscle stimulation for chronic heart failure: an alternative tool for exercise training? Curr Heart Fail Rep 2010; 7(2): 52-58.

9. Karavidas Al, Raisakis KG, Parissis JT, et al. Functional electrical stimulation improves endothelial function and reduces peripheral immune responses in patients with chronic heart failure. Eur J Cardiovasc Prev Rehabil 2006; 13(4): 592-597.

10. Maillefert JF, Eicher JC, Walker P, et al. Effect of low-frequency electrical stimulation of quadriceps and calf muscles in patients with chronic heart failure. J Cardiopulm Rehabil 1998; 18(4): 277-282.

11. Vaquero AF, Chicharro JL, Gil L, et al. Effects of muscle electrical stimulation on peak $\mathrm{VO}_{2}$ in cardiac transplant patients. Int J Sports Med 1998; 19(5): 317-322.

12. Harris S, LeMaitre JP, Mackenzie G, et al. A randomised study of home-based electrical stimulation of the legs and conventional bicycle exercise training for patients with chronic heart failure. Eur Heart J 2003; 24(9): 871-878.

13. Nuhr MJ, Pette D, Berger R, et al. Beneficial effects of chronic low-frequency stimulation of thigh muscles in patients with advanced chronic heart failure. Eur Heart J 2004; 25(2): 136-143.

14. Dobsak P, Novakova M, Siegelova J, et al. Low-frequency electrical stimulation increases muscle strength and improves blood supply in patients with chronic heart failure. Circ J 2006; 70: 75-82.

15. Dobsak P, Navakova M, Fiser B, et al. Electrical stimulation of skeletal muscles. An alternative to aerobic exercise training in patients with chronic heart failure? Int Heart J 2006; 47(3): 441-453.

16. Deley G, Eicher JC, Verges B, et al. Do low-frequency electrical myostimulation and aerobic training similarly improve performance in chronic heart failure patients with different exercise capacities? J Rehabil Med 2008; 40(3): 219-224.

17. Karavidas A, Parissis J, Arapi S, et al. Effects of functional electrical stimulation on quality of life and emotional stress in patients with chronic heart failure secondary to ischaemic or idiopathic dilated cardiomyopathy: a randomised, placebo-controlled trial. Eur J Heart Fail 2008; 10(7): 709-713.

18. Karavidas A, Parissis JT, Matzaraki V, et al. Functional electrical stimulation is more effective in severe symptomatic heart failure patients and improves their adherence to rehabilitation programs. J Card Fail 2010; 16(3): 244-249.

19. Banerjee $\mathrm{P}$, Caulfield $\mathrm{B}$, Crowe $\mathrm{L}$, et al. Prolonged electrical muscle stimulation exercise improves strength, peak $\mathrm{VO}_{2}$, and exercise capacity in patients with stable chronic heart failure. J Card Fail 2009; 15(4): 319-326.

20. Soska V, Dobsak P, Pohanka M, et al. Exercise training combined with electrostimulation in rehabilitation of patients with chronic heart failure: a randomized trial. Biomed Pap Med Fac Univ Palacky Olomouc Czech Repub 2012, dx.doi.org/10.5507/bp.2012.096.

21. Quittan M, Sochor A, Wiesinger GF, et al. Strength improvement of knee extensor muscles in patients with chronic heart failure by neuromuscular electrical stimulation. Artif Organs 1999; 23(5): 432-435.

22. Quittan M, Wiesinger GF, Sturm B, et al. Improvement of thigh muscles by neuromuscular electrical stimulation in patients with refractory heart failure: a single-blind, randomized, controlled trial. Am J Phys Med Rehabil 2001; 80(3): 206-214.

23. Sbruzzi G, Ribeiro RA, Schaan BD, et al. Functional electrical stimulation in the treatment of patients with chronic heart failure: a metaanalysis of randomized controlled trials. Eur J Cardiovasc Prev Rehabil 2010; 17(3): 254-260.

24. Smart NA, Dieberg G, Giallauria F. Functional electrical stimulation for chronic heart failure: a meta-analysis. Int J Cardiol 2012; 167(1): 80-86, doi:10.1016/j.icard.2011.12.019.

25. Neder JA, Sword D, Ward SA, et al. Home based neuromuscular electrical stimulation as a new rehabilitative strategy for severely disabled patients with chronic obstructive pulmonary disease (COPD). Thorax 2002; 57(4): 333-337.

26. Bourjeily-Habr G, Rochester CL, Palermo F, et al. Randomised controlled trial of transcutaneous electrical stimulation of the lower extremities in patients with chronic obstructive pulmonary disease. Thorax 2002; 57(12): 1045-1049.

27. Zanotti E, Felicetti G, Maini M, et al. Peripheral muscle strength training in bed-bound patients with COPD receiving mechanical ventilation. Chest 2003; 124(1): 292-296.

28. Vivodtzev I, Pepin J, Vottero G, et al. Improvement in quadriceps strength and dyspnea in daily tasks after 1 month of electrical stimulation in severely deconditioned and malnourished COPD. Chest 2006; 129(6): 1540-1548.

29. Dal Corso S, Napolis L, Malaguti C, et al. Skeletal muscle structure and function in response to electrical stimulation in moderately impaired COPD patients. Respir Med 2007; 101(6): 1236-1243.

30. Napolis L, Dal Corso S, Neder J, et al. Neuromuscular electrical stimulation improves exercise tolerance in chronic obstructive pulmonary disease patients with better preserved fat-free mass. Clinics 2011; 66(3): 401-406.

31. Abdellaoui A, Prefaut C, Gouzi F, et al. Skeletal muscle effects of electrostimulation after COPD exacerbation: a pilot study. Eur Respir J 2011; 38(4): 781-788.

32. Vivodtzev I, Debigare R, Gagnon P, et al. Functional and muscular effects of neuromuscular electrical stimulation in patients with severe COPD. Chest 2012; 141(3): 716-725.

Tables: 0

Figures: 0

References: 32

Received: 23.06.2016

Revised: 24.06.2016

Accepted: 28.06 .2016

Address for correspondence:

Ewa Kucio, PhD

Wydział Fizjoterapii AWF

ul. Mikołowska 72A

40-065 Katowice

Polska

Tel.: +48 692 704- 858

E-mail: ewakucio@poczta.fm 